\title{
Figure(s) du traducteur
}

"Figure(s) du traducteur". Curieux titre pour le deuxième numéro de Convergences francophones : vague et à la pluralisation incertaine, ce choix doit être expliqué, justifié et mis en adéquation avec celui de la photo de couverture de notre numéro. Qu'entend-on par figure(s)? La polysémie du terme ne semble guère des plus favorables à stabiliser cet objet d'étude. Toutefois son utilisation répétée dans la recherche en traduction (voir par exemple cet appel à contribution de 2013 ou plus récemment le nôtre) laisse voir que c'est peut-être cette souplesse terminologique qui sied le mieux à l'objet que l'on tente de cerner.

Depuis les "tournants" culturels et sociologiques (nous pensons à l'oeuvre de Bruno Latour et sa théorie de l'acteur-réseau), le traducteur, jusque là quelque peu délaissé par les chercheurs en traduction au profit de ses "productions", retrouve peu à peu une position centrale au sein des recherches en traductologie. Le traducteur cesse progressivement d'être considéré comme un simple "medium" transparent pour devenir un " médiateur culturel » (D'hulst) ou encore un créateur, c'est-à-dire le pivot essentiel du processus de traduction. Ces figures redécouvertes ont notamment fait l'objet d'études récentes portant le même titre que ce numéro: une anthologie dirigée par Elzbieta Skibinska parue en 2012 et le volume 19 de TTR paru en 2006.

C'est dans le sillage de ces travaux portant sur cet acteur de l'ombre et de son "apport... dans l'histoire intellectuelle et culturelle de l'humanité » (Deslisle et Woodsworth 18) que se place ce deuxième numéro de Convergences francophones. . L'illustration choisie pour la de couverture illustre notre démarche: la loupe, cachant partiellement le titre, tente de cerner cette figure souvent élusive. Si l'importance de son rôle dans notre société est indéniable, le traducteur, partiellement dans l'ombre de ses productions et des auteurs "originaux", perdu au sein de la chaîne de traduction (travaillant parfois avec un autre traducteur, un éditeur apportant des révisions plus ou moins majeures, un réviseur en chef, etc.), demeure une figure mal définie voire "invisible" (Venuti).

En jouant sur la polysémie de ce terme, ce volume propose de (re)découvrir cette figure essentielle à différentes époques, dans différents genres et dans diverses aires géographique. En l'observant à la loupe, nous serons peut-être mieux à même de comprendre et de (re)définir le rôle de cet acteur essentiel au sein de notre société.

La première contribution s'intéresse donc à cette figure de l'ombre lorsqu'elle se retrouve sous le feu des projecteurs. Dans leur contribution, Juan Zapata et Valérie Le Plouhinec proposent une étude sur le terrain d'un milieu largement inexploré: les prix de traduction littéraire en France. Leur approche résolument sociologique, ponctuée d'entretiens avec des figures importantes du domaine littéraire en traduction, propose d'explorer les rouages des prix de traduction. Au coeur de l'étude se joue encore une fois la question de l'invisibilité du traducteur et de la traduction et de son statut au sein du champ littéraire. 
L'article de Jean-Jacques Tatin Gourier examine dans un corpus varié les réflexions sur la traduction de Marie Joseph Chénier, auteur maintenant occulté par son frère, le poète André Chénier. A l'articulation des XVIIIe et XIXe siècles, les écrits de Marie Joseph Chénier montrent l'intérêt de ce dernier pour la traduction notamment d'oeuvres contemporaines et la revendication de leur importance dans le panorama littéraire qu'il établit. L'étude de textes divers (préface à ses propres traductions, écrits politiques, histoire littéraire) met ainsi en évidence la figure d'un médiateur culturel, soucieux de la nécessité de faire reconnaître le rôle du traducteur dans la société des arts et et des lettres. Elle souligne également la fonction de refuge que peut revêtir la traduction pour un homme de lettres face à la censure grandissante, ici du Consulat et de l'Empire.

Interrogeant le transfert d'oeuvres du corpus français vers une autre aire linguistique, François Bouchard s'attache à mettre au jour les processus textuels et visuels à l'oeuvre dans la traduction faite par Carlo (Lorenzini) Collodi d'un recueil rassemblant des contes de Charles Perrault, de Mme d'Aulnoy et de Mme Leprince de Beaumont. Le futur auteur des Aventures de Pinocchio se révèle fin manipulateur : sous la patte du traducteur, les caractéristiques des trois auteurs français finissent par disparaître, laissant voir un ensemble homogénéisé, où, comme le note Bouchard, "il ne reste pour tout auteur que le traducteur".

Frédéric le Gouriérec s'intéresse à tout un champ d'étude, la sinologie, à travers l'un de ses pères fondateurs en France: Jean-Pierre Abel-Rémusat. Véritable cas d'école en études de la traduction, les réflexions d'Abel-Rémusat sur le chinois traduisent certains préjugés et stéréotypes sur la langue toujours d'actualité. Ce qui devrait être une "simple" grammaire a donné lieu à diverses dérives métaphysiques au sein des discours sinologiques contemporains, traces toujours bien visibles de l'impact d'un traducteur-créateur d'un champ d'étude.

A l'instar de la contribution précédente, Anne-Claire Collier analyse la traduction/réception en France de tout un champ d'études : les études postcoloniales. Méconnues jusqu'en 2005, ce courant de pensée fait son entrée sur le territoire hexagonal à travers deux maisons d'édition aux profils bien distincts : Payot et Amsterdam. Le choix du traducteur ou de la traductrice s'avère déterminant dans la réception critique des textes traduits pour qui ce choix dénote une certaine éthique intellectuelle, gage d'autorité. Si les approches à la traduction des ouvrages majeurs de ce courant de pensée diffèrent selon les maisons d'édition, leur utilisation du label "colonial" ou "postcolonial" manifeste un désir de regrouper diverses contributions sous le même courant de pensée afin de le rendre accessible à un plus large public. L'intérêt (voire la collusion) du monde universitaire, littéraire et politique pour ces études démontre une volonté de comprendre le malaise social français actuel.

Finalement, Chantal Ringuet s'attache à l'étude du yiddish à Montréal. Si cette langue et sa culture sont encore peu étudiées, elles représentent néammoins un domaine de recherche en pleine expansion. Après un rappel des particularités de la langue et de la culture yiddish et de son impact culturel au Canada, l'auteur retrace son travail minutieux de traductrice du yiddish. Des difficultés du choix des textes à l'absence d'école de traduction du yiddish en français québécois, 1'“archéologue textuelle" a pour mission d'enrichir le paysage littéraire québécois. 
Nous tenons à vivement remercier les auteurs de ce numéro ainsi que ceux, nombreux, ayant répondu à notre appel à contributions. Pour leur soutien, nous tenons aussi à remercier Amr Ahmed, Paul Bandia, Hélène Buzelin, Jean Delisle, Evelyn Dueck, Patricia Godbout, Lieven D'hulst, Sarah Harvey, Nathalie Heinich, Claire Huot, Evelyn Kee Mew, Leyla Moharreri, Greg Robinson, Sherry Simon.

Les éditeurs 


\section{Bibliographie}

Buzelin, Hélène. "Independent Publishers in the Networks of Translation. » $T R$ : traduction, terminologie, rédaction 19.1 (2006):135-73.

D'hulst, Lieven. Essais d'histoire de la traduction. Avatars de Janus. Paris: Classiques

Garnier, 2014.

Figure(s) du traducteur. Ed. Elżbieta Skibińska. Wrocław: Romanica Wratislaviensa, 2012.

«Figures du traducteur/Figures du traduire I. » Dir. Gillian Lane-Mercier. TTR : traduction, terminologie, rédaction 19.1 (1998).

Les traducteurs dans l'histoire. Eds. Jean Delisle et Judith Woodsworth. Les presses de

l'université d'Ottawa, 1995.

Venuti, Lawrence. The Translator's Invisibility. A History of Translation. London/New York:

Routledge, 1995.

Note

La tenue en avril 2015 d'une journée d'étude sur le même thème fera l'objet d'une publication d'actes dans le numéro 2.2 de la revue à paraître à l'automne 2015. 